\title{
Aplicación de la caracterización eléctrica al estudio de las fases hidratadas de cemento con adición de escorias de centrales térmicas
}

\author{
E. MENÉNDEZ ${ }^{1}$, J. DE FRUTOS ${ }^{2}$, A. M. ALVARO \\ 'IETcC-CSIC. C/ Serrano Galvache 4, 28033. Madrid \\ 2POEMMA-CEMDATIC. ETSI Telecomunicación-UPM. Avda Complutense, 30. 28040 Madrid
}

\begin{abstract}
En este trabajo, se estudia la evolución desde su inicio de las fases de hidratación de materiales cementicios a los que se incorporan cenizas volantes y escorias procedentes de centrales térmicas. Mediante medidas de espectroscopía de impedancia eléctrica (EIS), y de difracción de rayos X (XRD), se analiza el proceso desde la etapa inicial visualizando los cambios que se producen. Se consideran diferentes elementos de sustitución y se determina para cada uno de ellos cuando se inician los procesos, cuando se completan y los cambios que sufren durante el mismo. Se relacionan los valores de EIS con los de DRX para determinar las fases que se transforman, y se indica para cada sustitución como se modifica la transformación de las fases en función del tiempo y en comparación con el material de referencia. Se pone de manifiesto que la utilización de EIS, trabajando en función de la frecuencia y con los parámetros eléctricos adecuados es una herramienta muy potente para hacer el seguimiento a tiempo real de estos procesos.
\end{abstract}

Palabras clave: ceniza de fondo, propiedades químicas, microestructura, proceso de hidratación.

Application of the electrical characterization to the study of the hydrated phases of the cement with coal bottom ash

The present paper investigates the influence of using Bottom and Fly Ash as partial replacement of cement in the hydration process. Through measurements of electrical impedance spectroscopy (EIS) and X -ray diffraction (XRD), we analyze from the early stages to the hydration process to the end. Values of EIS, XRD and its relation, are used to determine transformation of hydrated phases, and for each of the substitutions, is indicated as modified the hydrated phase as a function of time and compared it with the reference material. It also proves the relevance of using EIS measures in real time, and as nondestructive testing to characterize the hydration process of these materials.

Keywords: bottom ash, chemical properties, microstructure, electrical characterization, hydration process.

\section{INTRODUCCIÓN}

Uno de los grandes problemas medioambientales asociados a las centrales térmicas a nivel mundial, es el aumento de los residuos totales, especialmente cuando el carbón que se utiliza como combustible es de baja calidad, no sólo por el problema de almacenamiento de dichos residuos, sino de manera especial por los problemas medioambientales y de salud. Los residuos producidos se dividen en cenizas volantes ((FA) del inglés Fly Ash) y escorias ((BA), del inglés Bottom Ash). Aproximadamente, el $80 \%$ de estos residuos corresponden a las FA, que están constituidas por partículas finas que permanecen en suspensión en las corrientes de aire. Por su parte, las BA corresponden aproximadamente al $20 \%$ de estos residuos, y están formados por partículas grandes que se depositan en la base del horno, y que son debidas a restos de fusión, aglomerados, vitrificados [1-2]. Ambos tipos de residuos contienen trazas metálicas que pueden diluirse en agua y contaminar los ecosistemas acuáticos [3]. En 2009 en Europa, se generaron más de 41 millones de toneladas de cenizas y escorias de carbón, y de estos, aproximadamente la mitad se arrojaron a vertederos [4]. Esto genera un importante problema medioambiental al que se le están buscando soluciones con aprovechamiento de mejora en diferentes sectores. La utilización de las FA como adiciones a materiales en base cemento ((SMC) Supplementary Cementitious Material) se presenta como una potencial aplicación alto valor añadido de los mismos y de alto interés medioambiental [5] que podría mejorar las propiedades del cemento, y reduciría tanto el consumo de materias primas como la emisión de $\mathrm{CO}_{2}$ en el proceso de fabricación del cemento.

Por el contrario, las BA hasta el momento no han sido considerados como potenciales SMC con alto valor añadido en la industria de la construcción. El BA, o bien se elimina o bien se utiliza como sustituto de bajo costo para los áridos o arena en la producción de bloques de hormigón, o en otros países, como sustrato en la construcción de carreteras [1-2]. Sin embargo, este punto de vista ha ido cambiando en los últimos 
años. Cheriaf et al. [6] han evidenciado la actividad puzolánica del BA con cal a los 28 días y han sugerido el uso de BA como adición activa en materiales cementicios. Este hecho ha sido también observado por Jaturapitakkul et al. [7] y también por Kurama y Kaya [8], que han llegado a la conclusión de que la adición de BA como sustitución parcial del cemento hasta niveles de un $10 \%$, podría mejorar las propiedades mecánicas del hormigón. Ha habido diferentes experimentos mezclando BA con otras adiciones. Así, Kula y col. [9] han encontrado que la sustitución de cemento Portland (PC) por residuos BA y FA de colemanita, da lugar generalmente a un mejor rendimiento en la resistencia a la compresión de la pasta de PC. Kula y col. [10] También estudiaron el uso de residuos de mineral tincal $\left(\mathrm{Na}_{2} \mathrm{~B}_{4} \mathrm{O}_{7} \cdot 10 \mathrm{H}_{2} \mathrm{O}\right)$ en el cemento y encontraron que el uso de $\mathrm{BA}$ como reemplazo parcial de residuos de tinkal permite obtener un mejor rendimiento del hormigón que con el remplazo por FA. Por su parte, Canpolat et al. [11] estudiaron los efectos de la sustitución de la zeolita y BA o FA en diferentes proporciones en PC, comprobando que la mayoría de los cementos estudiados mostraron una resistencia a la compresión mayor que el cemento CEM I 42.5. Recientemente, Argiz et al. [12] han estudiado el efecto sobre las propiedades físicas y mecánicas de los morteros con diferentes proporciones de FA y BA como reemplazo parcial del cemento hasta un $35 \%$ y han llegado a la conclusión de que no hay diferencias tecnológicas entre ambas cenizas. A pesar de estas investigaciones, las normas europeas no permiten el uso como la adición del BA al cemento.

En los materiales cementicios, el proceso de hidratación es un factor determinante que influye en la microestructura final del material y como consecuencia en sus propiedades mecánicas y en su durabilidad. Durante este proceso, se forman silicatos de calcio hidratados (CSH) e hidróxido cálcico (portlandita). El CSH es parte constituyente de la matriz del cemento y la portlandita forma parte tanto de la matriz como de la estructura de poros del mismo. Además, la reacción de los aluminatos y ferritoaluminatos, en presencia de yeso, da lugar a la formación de etringita (C6AS3 H32) que, en una segunda etapa y en defecto de azufre, puede reaccionar con el aluminato para formar monosulfoaluminato 3C4ASH12, apareciendo estos compuestos tanto en la matriz como en las zonas porosas del material. Por su parte, la hidratación de los cementos con adiciones requiere el estudio de los mecanismos de hidratación tanto del cemento Portland, como de cada una de las adiciones, así como el proceso de interacción.

El uso de SMC, al introducir fases diferentes, implica procesos de hidratación más complejos [13]. En el caso de cementos con sustitución parcial de FA, los productos de hidratación son fundamentalmente los mismos que para el cemento [14], pero la reacción cinética asociada al proceso de hidratación se modifica [15]. Son necesarios estudios de los mecanismos de hidratación del cemento para cada uno de los SCM, así como del proceso de interacción entre ellos, para comprender el proceso de hidratación y en consecuencia la formación del material.

Los procesos de hidratación son fuertemente exotérmicos, fundamentalmente en las primeras etapas, por lo que tradicionalmente, se han estudiado usando el método de calorimetría semi-adibática. Con esta técnica, mediante la medida de la liberación de calor, se identifican los diferentes procesos y el mecanismo de hidratación asociado. Esta técnica presenta carencias importantes [16], tales como que el contenido de calor liberado, no mantiene una relación de proporcionalidad con el grado de hidratación del cemento, y no permite ni tan siquiera estimar las propiedades físicas del mismo. Este método sólo proporciona una aproximación en el conocimiento de la hidratación del cemento y deja espacio para que los investigadores exploren formas más precisas para entender el proceso.

La Espectroscopia de Impedancia Eléctrica (EIS), es un método no destructivo, que se ha utilizado recientemente para estudiar el proceso de hidratación de los cementos [17-23]. Tradicionalmente, EIS se ha utilizado para la caracterización de materiales funcionales, fundamentalmente electrocerámicas [24]. En el campo de los cementos, se utilizó para estudiar las propiedades microestructurales de los morteros de cemento endurecido con el fin de evaluar la influencia de la relación agua/cemento [25-29], de diferentes adiciones [25; 30-32], de la temperatura de curado [33] o del grado de deterioro del material [26, 31].

Químicamente, la hidratación del cemento implica la disolución de iones y la formación de nuevos compuestos químicos. Físicamente, este proceso origina la formación de la microestructura y el proceso de disminución de la porosidad. Cuando se añade agua al cemento los iones solubles se disuelven en el agua. La solución de iones en los poros es conductora. La conductividad depende en estas condiciones de la concentración de iones en la solución y de la microestructura, especialmente de la porosidad y de la conectividad entre poros en la pasta de cemento. Por esta razón, la determinación de los parámetros eléctricos de una mezcla de cemento, puede utilizarse para interpretar los procesos de hidratación [20].

Menéndez et al han realizado una serie de trabajos relacionados con los procesos de hidratación en las primeras etapas del proceso, mediante medidas de Espectroscopía de Impedancia Eléctrica (EIS), relacionando la respuesta con las frecuencias de análisis, lo que se complementa con métodos tradicionales [20-22]. Estos autores establecieron como condiciones óptimas de medida la realización de ciclos en el rango de $1 \mathrm{~Hz}$ a $1 \mathrm{MHz}$, barriendo en escala logarítmica y con 10 puntos por década.

En este trabajo se estudia la influencia de la sustitución parcial de BA, TA y combinaciones de ambas en el cemento en los procesos de hidratación mediante medidas de EIS, DRX y SEM. Se analizan los cambios tanto en los tiempos como en las fases de hidratación y se compara el resultado para los diferentes tipos de sustitución.

\section{EXPERIMENTAL}

\subsection{Caracterización de las cenizas y escorias del carbón}

Para este estudio, se han seleccionado dos plantas térmicas españolas que utilizan carbones de calidades diferentes. Denominaremos a las plantas con las letras " $\mathrm{C}$ " y " $\mathrm{L}$ ". Las primeras (C), trabajan con carbón mezcla, $90 \%$ procedente de Sudáfrica con un $10 \%$ procedente de Colombia. Por su parte, las denominadas L, utilizan carbón español de menor calidad, y que en las cenizas volantes presenta mayor proporción de materia orgánica. Se han seleccionado residuos FA y BA en ambas plantas, por lo que disponemos de cuatro tipos de residuos para su estudio, que denominaremos FAC y BAC 
TABLA I. COMPOSICIÓN QUíMICA DEL CEMENTO I, DE LAS CENIZAS VOLANTES (FA) Y DE LAS ESCORIAS (BA)

\begin{tabular}{|c|c|c|c|c|c|c|c|c|c|c|c|c|c|c|c|c|c|c|c|c|c|}
\hline & \multicolumn{2}{|c|}{$\mathrm{SiO}_{2}$} & \multirow[b]{2}{*}{$\frac{0^{m}}{z^{n}}$} & \multirow[b]{2}{*}{$0_{\mathcal{L}^{N}}^{m}$} & \multicolumn{2}{|c|}{$\mathrm{CaO}$} & \multirow{2}{*}{ 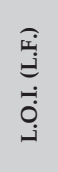 } & \multicolumn{14}{|c|}{ Minoritarios } \\
\hline & & 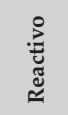 & & & & 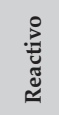 & & $\stackrel{O^{N}}{=}$ & $\sum_{\sum}^{\circ}$ & $\begin{array}{l}0^{1 n} \\
2^{n}\end{array}$ & $\stackrel{0}{\mathfrak{o}^{\prime}}$ & O) $^{m}$ & के & $\begin{array}{l}0 \\
\tilde{Z}^{N}\end{array}$ & $\stackrel{\stackrel{D}{N}^{N}}{ }$ & $\stackrel{0}{\stackrel{\Xi}{\Sigma}}$ & $\begin{array}{l}0^{m} \\
>^{n}\end{array}$ & $\begin{array}{l}0^{m} \\
3^{n}\end{array}$ & 옳 & $\dot{U}$ & O \\
\hline CEM I & 20,05 & 0,00 & 5,19 & 2,20 & 62,04 & 0,00 & 2,51 & 0,24 & 3,61 & 0,14 & 0,86 & 2,83 & 0,00 & 0,30 & 0,00 & 0,00 & 0,00 & 0,00 & 0,00 & 0,03 & 0,00 \\
\hline FAC & 50,68 & 25,33 & 28,85 & 5,11 & 6,01 & 5,59 & 3,63 & 1,62 & 1,44 & 1,11 & 0,54 & 0,40 & 0,22 & 0,18 & 0,06 & 0,04 & 0,04 & 0,03 & 0,02 & 0,01 & 0,01 \\
\hline FAL & 46,21 & 25,66 & 28,97 & 4,01 & 6,05 & 2,48 & 9,14 & 0,97 & 1,51 & 0,99 & 0,61 & 0,70 & 0,22 & 0,26 & 0,05 & 0,05 & 0,21 & 0,02 & 0,02 & 0,01 & 0,01 \\
\hline BAC & 51,59 & 25,22 & 27,39 & 6,28 & 7,58 & 6,10 & 1,85 & 1,61 & 1,37 & 1,03 & 0,47 & 0,16 & 0,23 & 0,15 & 0,06 & 0,06 & 0,03 & 0,04 & 0,09 & 0,00 & 0,01 \\
\hline BAL & 53,19 & 29,12 & 21,41 & 9,86 & 7,09 & 2,64 & 1,55 & 1,10 & 1,98 & 0,54 & 1,56 & 0,11 & 0,18 & 0,78 & 0,04 & 0,09 & 0,04 & 0,32 & 0,01 & 0,01 & 0,13 \\
\hline
\end{tabular}

TABLA II. COMPOSICIONES CONSIDERADAS

\begin{tabular}{|c|c|c|c|c|c|c|c|}
\hline Tipo de Cemento UNE/EN 197-1 & CEM I & FAC & BAC & FAL & BAL & Relación a/c & Nombre de la mezcla \\
\hline \multirow{2}{*}{ CEM I } & 100 & 0 & 0 & 0 & 0 & 0,5 & PI-REF \\
\hline \multirow{5}{*}{ CEM II/B } & 75 & 25 & 0 & 0 & 0 & 0,5 & FAC-25 \\
\cline { 2 - 9 } & 75 & 20 & 5 & 0 & 0 & 0,5 & FBAC-25 \\
\cline { 2 - 9 } & 75 & 0 & 25 & 0 & 0 & 0,5 & BAC-25 \\
\cline { 2 - 8 } & 75 & 0 & 0 & 25 & 0 & 0,5 & FAL-25 \\
\cline { 2 - 8 } & 75 & 0 & 0 & 20 & 5 & 0,5 & FBAL-25 \\
\cline { 2 - 8 } & 75 & 0 & 0 & 0 & 25 & 0,5 & BAL-25 \\
\hline
\end{tabular}

cuando se obtienen de centrales térmicas C, y FAL y BAL si provienen de centrales térmicas $\mathrm{L}$.

\subsubsection{ANÁLISIS QUÍMICO}

La composición química de las cenizas y las escorias se ha estudiado haciendo uso de fluorescencia de Rayos $X$ (equipo Bruker S8 Tiger). La cantidad de materia perdida por combustión (LOI, del inglés "Lost on Ignition") se determina haciendo uso del método descrito en la norma UNE/EN 196-2 [34] dónde los residuos se queman durante una hora. El contenido total de $\mathrm{CaO}$ reactivo se determina de acuerdo con la norma UNE/EN 196- 2 [34]. Los componentes principales se recogen en la Tabla I.

En general, la FA y la BA para cada central térmica tienen una composición similar excepto en el contenido de calcio, de hierro, magnesio y cromo para los que es claramente mayor.

La Norma Europea UNE/EN 197-1 [35], establece un máximo en el LOI en cenizas volantes (y por extensión en escorias) del $9 \%$, para evitar posibles problemas de durabilidad. El contenido de LOI en todos los residuos utilizados es inferior a este límite excepto en el caso de las $F A B$, en las que es ligeramente superior. Este relativamente alto valor de LOI puede explicarse por la baja calidad del carbón quemado en la central térmica L. Por su parte, las muestras BAL presentan un contenido similar de LOI al de las BAC, y siempre inferior a las FAL examinadas.

Las cenizas volantes, se clasifican en dos grupos en función del contenido en peso del $\mathrm{CaO}$ reactivo [35]. Si el contenido es inferior al $10 \%$ se les denomina silícicos y si superan el $10 \%$, se les denomina calcáreos. De acuerdo con esto, las cenizas volantes estudias son silícicas, y por analogía, las escorias son también silícicas. No obstante, el nivel de $\mathrm{CaO}$ reactivo es considerablemente mayor tanto para las cenizas como para las escorias en las centrales L que en las C.

\subsection{Caracterización de los procesos de hidratación en las mezclas}

Con el objetivo de estudiar los efectos de las diferentes adiciones en los procesos de hidratación, se han preparado muestras de diferentes mezclas de los residuos con cemento de tipo I y posteriormente se han estudiado mediante Difracción de Rayos X (XRD) Microscopía Electrónica de Barrido (SEM) y Espectroscopía de Impedancia Eléctrica (EIS).

\subsubsection{EL MATERIAL}

Se han preparado compuestos con una sustitución de un $25 \%$ de residuos (cenizas y/o escorias). Este porcentaje en la sustitución corresponde a los denominados CEM II/B por la norma [35]. Las mezclas se han preparado a partir de cemento CEM I 42.5 y en todos los casos se ha trabajado con una relación agua/ cemento del 0,5. En la tabla II se indica para cada mezcla la sustitución considerada, y la denominación asignada.

Para determinar el tipo de reacción química que tiene lugar en el material durante el proceso de hidratación, se ha analizado la evolución con el tiempo por diferentes técnicas. Las medidas de XRD se han realizado con un equipo Bruker $\mathrm{D} 8$, con ánodo de $\mathrm{Cu}$ y 2,2 kW, generador de Rayos $\mathrm{X}$ de 3 $\mathrm{kW}$, tensión de 20-60 kV y corriente 5-80 mA, goniómetro $\theta / \theta$ y detector ultrarrápido LYNXEYE.

Durante las primeras 24 horas de hidratación, se adquieren medidas en el rango de $2 \theta$ de 15 a 35 en ciclos repetidos cada 9 minutos. 


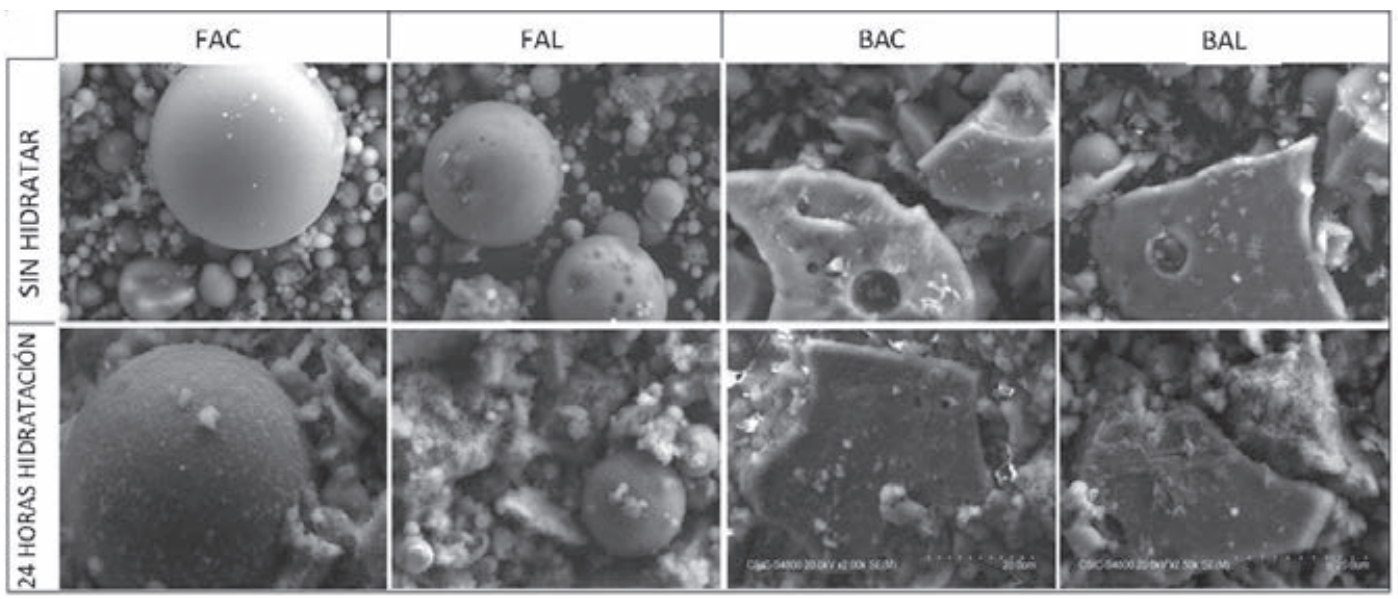

Figura 1. Micrografía SEM de las escorias y las cenizas volantes sin hidratar y tras 24 horas de hidratación.

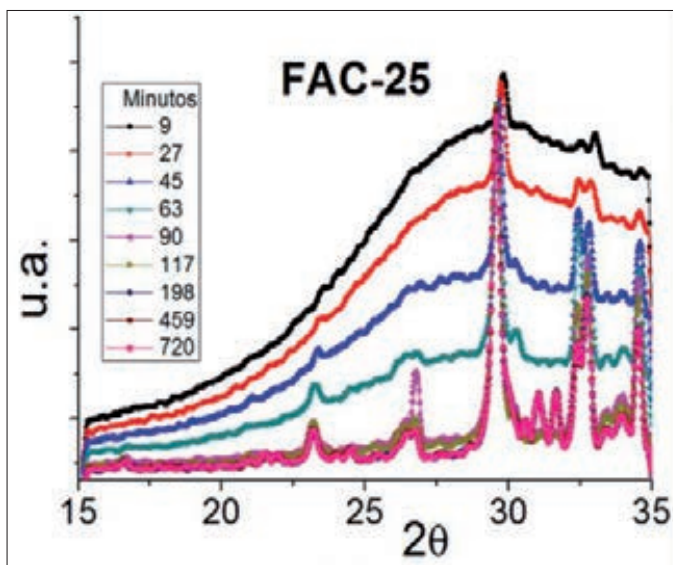

(a)

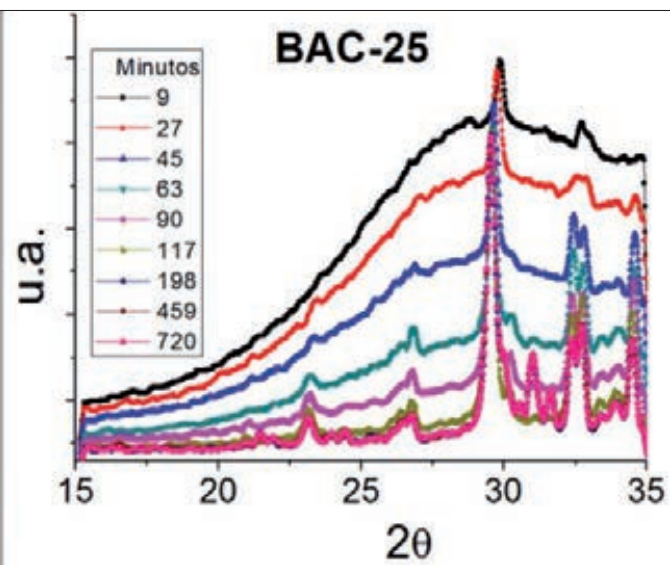

(b)

Figura 2. Seguimiento en el tiempo del proceso de hidratación mediante DRX, (a) FAC y (b) BAC.

Para las medidas eléctricas se aplica el método implementado por Menéndez et al [20-22]. Durante la primera hora de hidratación, se realizan medidas continuas de ciclos en el rango de $1 \mathrm{~Hz}$ a $10^{6} \mathrm{~Hz}$ (obteniendo un ciclo cada 4 minutos), y a partir de la primera hora, el rango se amplía entre $0,05 \mathrm{~Hz}$ a $10^{7} \mathrm{~Hz}$ (obteniéndose un ciclo cada 9 minutos). Las medidas de impedancia se han realizado haciendo uso de un analizador Solartron 1260A complementado con el módulo de medidas dieléctricas 1296 A. Esto permite el análisis en el rango de frecuencias de $10^{-6}$ a $10^{7} \mathrm{~Hz}$ y de impedancias de hasta $10^{17} \Omega$. Los datos se obtienen en ciclos continuos en los dos rangos de frecuencias indicados anteriormente, con barridos logarítmicos tomando diez puntos por década. Para el tratamiento de los datos se hace uso del software comercial ZView, y de software específico desarrollado por los autores.

\subsubsection{ANÁLISIS MICROESTRUCTURAL}

La microestructura tanto de las cenizas como de las escorias, se ha analizado mediante SEM. En la figura 1 se recogen las imágenes correspondientes a las adiciones básicas previas a la hidratación y tras 24 horas de hidratación. Puede observarse que mientras las FAS aparecen en su mayoría como partículas esféricas de diámetro inferior a $30 \mu \mathrm{m}$, las BAS tienen una geometría irregular y de mayor tamaño. Esta diferencia es debido a la forma diferente de generarse cada una de ellas. Después de 24 horas de hidratación, se observan las estructuras cristalinas características del proceso de hidratación, con resultados ligeramente diferentes para cada uno de los compuestos considerados.

\subsubsection{DIFRACCIÓN DE RAYOS X}

Se ha monitorizado la evolución de las diferentes composiciones en la etapa de hidratación, mediante DRX y EIS. Las medidas de DRX ponen de manifiesto la transformación de fases cristalinas iniciales por el efecto de hidratación del material. Como puede verse en la figura 2, hay un proceso de cambio muy rápido en la primera hora, presentando posteriormente una estructura cristalina con ligeros cambios hasta completar la hidratación. Este proceso se manifiesta de manera más acusada en el caso de las muestras con sustitución que las del material de referencia.

Se observa (figura 3) que en el proceso de hidratación, aunque aparecen fases diferenciadas para cada uno de los sistemas, la estructura base de los elementos de hidratación aparece en todos ellos.

Para determinar la evolución con el tiempo, se integra para los diferentes picos de difracción un ancho fijo, y se representa este valor para todo el rango de tiempos. Este estudio se ha realizado para todos los compuestos, determinando comportamientos similares pero con tiempos 

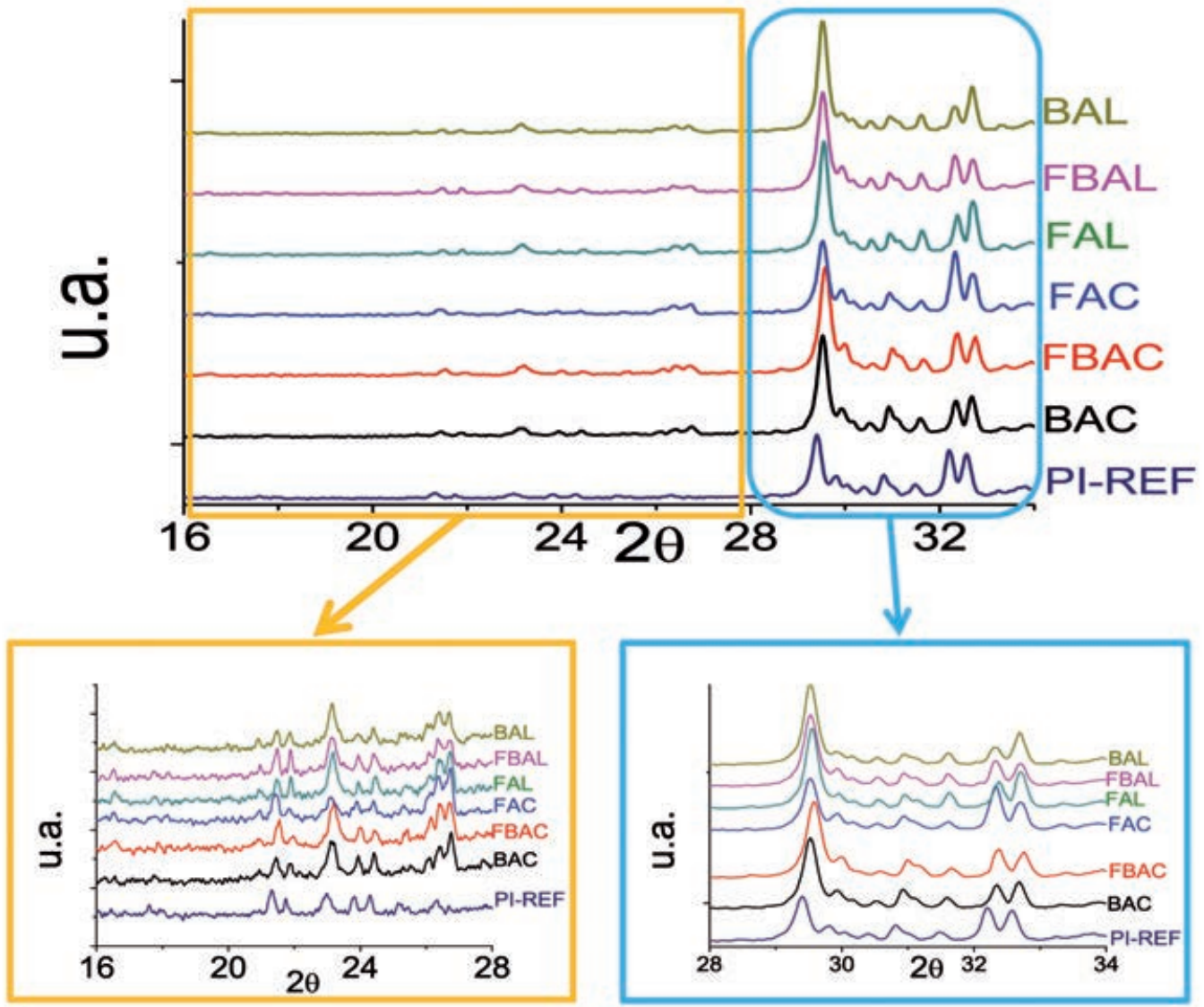

Figura 3. Espectros de DRX de todos los sistemas, después de 720 minutos desde el inicio del proceso de hidratación. En los recuadros inferiores se amplía la señal correspondiente a los rangos $2 \theta$ de 15 a 28 y de 28 a 35 respectivamente.

de transformación diferentes. La evolución de los diferentes picos con la transformación de fases cristalinas iniciales mediante el proceso de hidratación puede verse en la figura 4a. En ella se recoge la evolución de los picos más significativos para el material de referencia. El seguimiento de cada uno de los picos puede ayudar a determinar el momento en que el material alcanza su estado de hidratación. En las figuras $4 b$ y $4 \mathrm{c}$ se representa la evolución comparativa para todos los compuestos para dos picos concretos que permiten ver los tiempos de transformación como resultado de la hidratación para cada uno de ellos. El pico centrado en la posición $2 \theta$ de 30,96 muestra una transformación muy rápida que tiene lugar primero para el FAL25, prácticamente en la primera hora de hidratación, le sigue el BAC25, que se retrasa aproximadamente 25 minutos, y prácticamente una hora después tiene lugar en BAL25, PI-Ref, FAC25, FBAL, y por último FBAC. Aunque de forma menos marcada, lo mismo se observa en la figura $4 \mathrm{c}$ correspondiente al $2 \theta$ de 34,34 . En todos los casos la rampa de transición para 30,96, corresponde con el mínimo de relajación para el 34,34. Las flechas en trazos para la muestra de FAL25 y para el FBAC así lo reflejan.

\subsubsection{ESPECTROSCOPÍA DE IMPEDANCIA ELÉCTRICA}

De forma análoga, se ha realizado el estudio de la respuesta eléctrica de los diferentes compuestos a en función de la frecuencias y en un amplio rango de tiempo. En este caso, en las etapas iniciales se comporta como un conductor, pasando

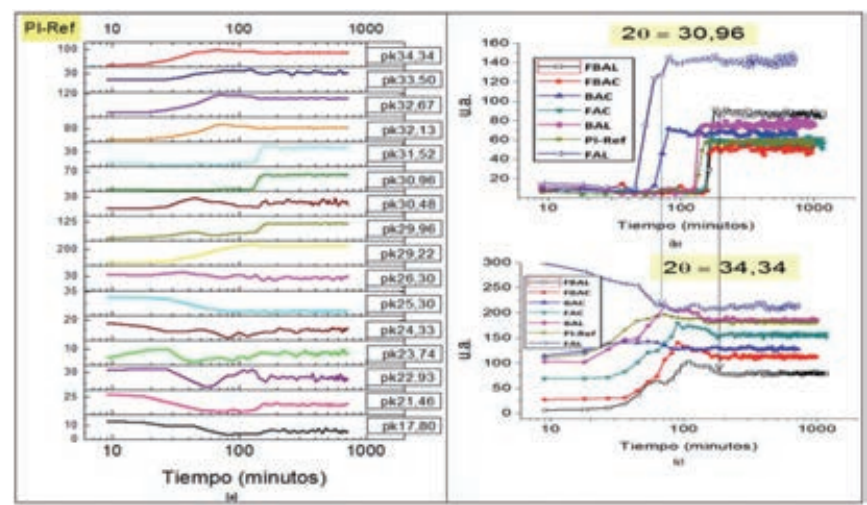

Figura 4. Evolución de los picos de DRX con el tiempo de hidratación. (a) representación de la evolución de los picos más relevantes para el PI-Ref, (b) y (c) representan la evolución con el tiempo para todas las composiciones de los picos de 30,96 y 34,40 respectivamente.

a tener un comportamiento dieléctrico una vez completado el proceso de hidratación. En la figura 5 se pone de manifiesto este comportamiento. En las figuras $5 a, 5 b$ y $5 c$ se representan los valores de la parte imaginara de la impedancia frente a la parte real para un rango de tiempos entre 9 minutos y 1250 minutos. Hasta tiempos superiores a los 450 minutos el comportamiento es semiconductor, y por encima de esos tiempos empieza a tener un comportamiento dieléctrico. 


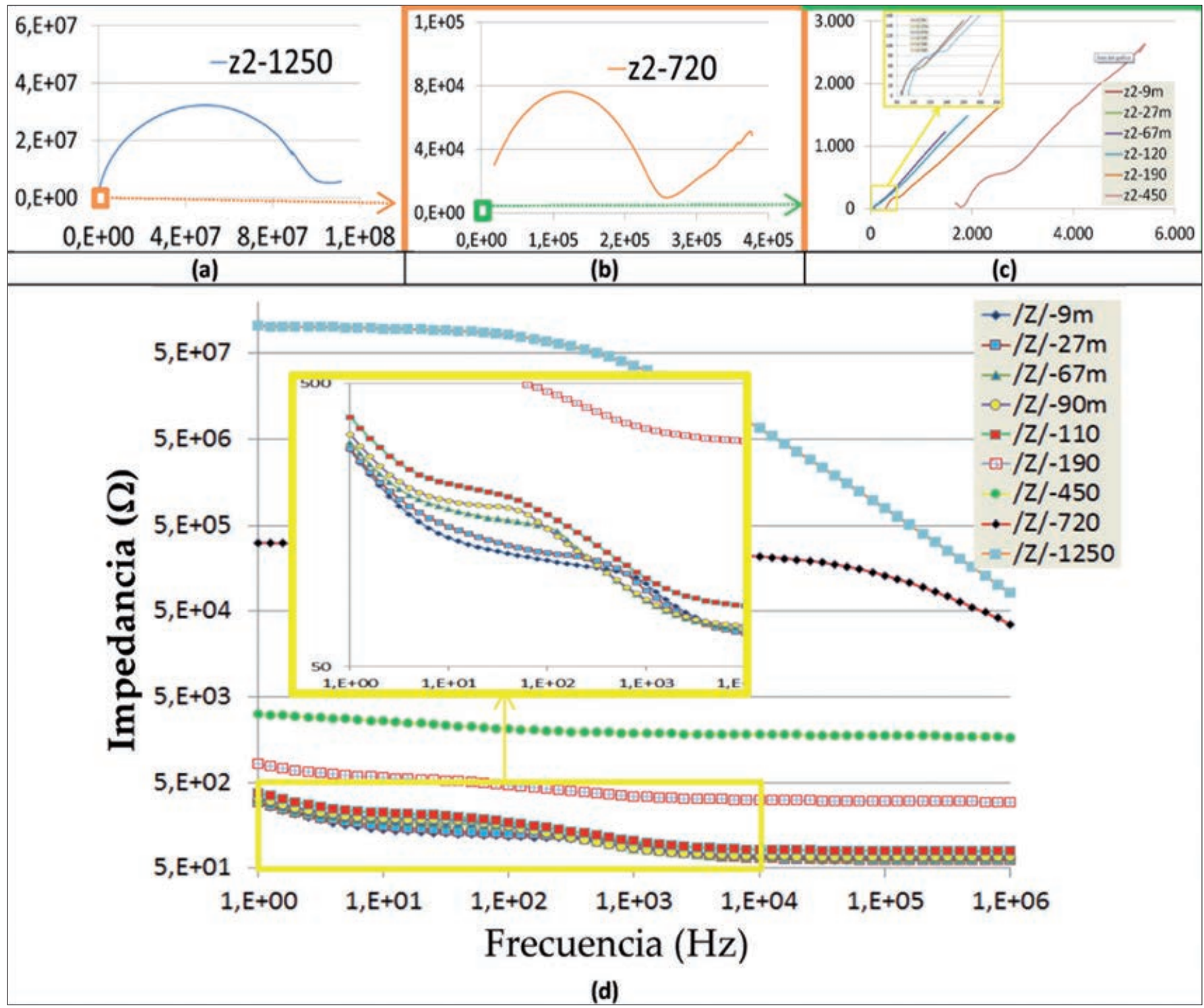

Figura 5. Diagramas de Nyquist del material de referencia (PI-Ref), para tiempos (a) 1250 minutos, (b) 720 minutos y (c) 450, 190, 120, 67, 27, 9 minutos, y (d) valor de impedancia en función de la frecuencia para los mismos tiempos.

Como puede verse también en la figura $5 \mathrm{~d}$, los valores de impedancia se incrementan en más de cinco órdenes de magnitud entre los inicios de la hidratación y los primeros 1250 minutos. Adicionalmente, el comportamiento eléctrico varía de forma notable con un cambio en la respuesta para tiempos del orden de los 450 minutos.

Para analizar la respuesta con el tiempo, se han analizado diferentes parámetros eléctricos. Al tener inicialmente un comportamiento conductor, estas variaciones se visualizan mejor con el módulo de la admitancia o con las pérdidas dieléctricas, mientras que para tiempos elevados, cuando el material se convierte en dieléctrico, la visualización es más clara haciendo uso de la impedancia eléctrica. En la figura 6 se representan para la muestra de referencia, conjuntamente los valores de módulo de admitancia y de la parte real de la impedancia, en función del tiempo de hidratación, y para diferentes frecuencias de análisis. Las bajas frecuencias resaltan los fenómenos asociados con la movilidad de carga asociados al proceso de hidratación, mientras que las altas frecuencias resaltan los fenómenos asociados a la conformación estructural del material. Así, en la figura 6a, se observa como inicialmente aumenta ligeramente el módulo de la admitancia (la conductividad del material) debido fundamentalmente a la descomposición de las fases del Clinker y en la disolución de los diferentes iones por la presencia del agua. Con el inicio de las siguientes fases, se bloquea la presencia de iones en el material, disminuyendo sustancialmente el valor de $\mathrm{Y}$. Si nos fijamos en la respuesta en frecuencia, podemos ver que mientras la transición de inicio de las fases debidas a la hidratación tiene lugar de forma brusca y muy pronunciada para altas frecuencia $\left(10^{5}\right.$ y $10^{6} \mathrm{~Hz}$, e incluso en $\left.10^{4}\right)$, lo que pone de manifiesto el cambio en la estructura interna del material con el cambio en la fases cristalinas, por su parte, para baja frecuencias, el proceso es prácticamente continuo, indicando el trasvase de iones de una estructura cristalina a otra. Si hacemos uso de la figura $6 \mathrm{~b}$, se potencia el resultado del material para tiempos de hidratación grandes. Es muy significativo el cambio de comportamiento que muestra a altas frecuencias (de nuevo $10^{5}$ y $10^{6} \mathrm{~Hz}$, e incluso en $10^{4}$ ). Después de un fuerte incremento de impedancia, que podemos 


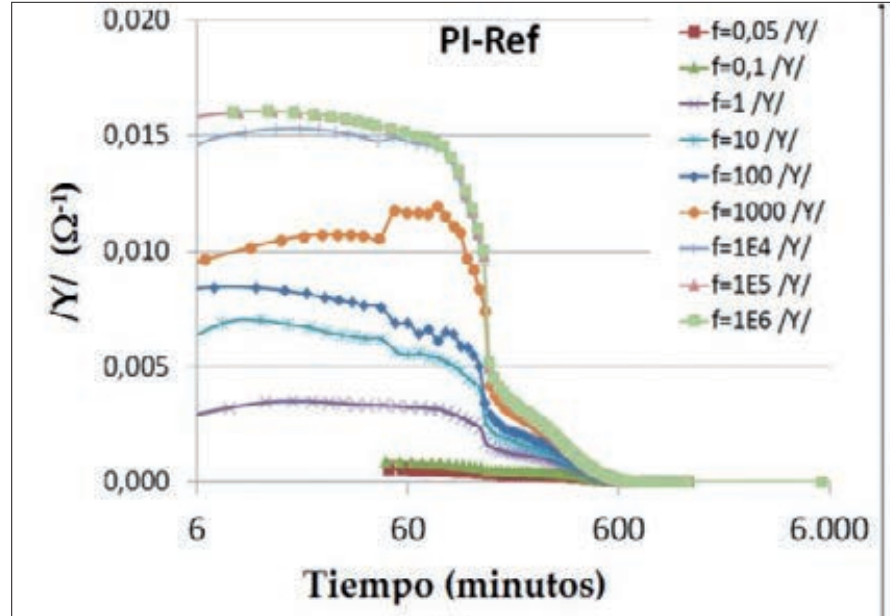

(a)

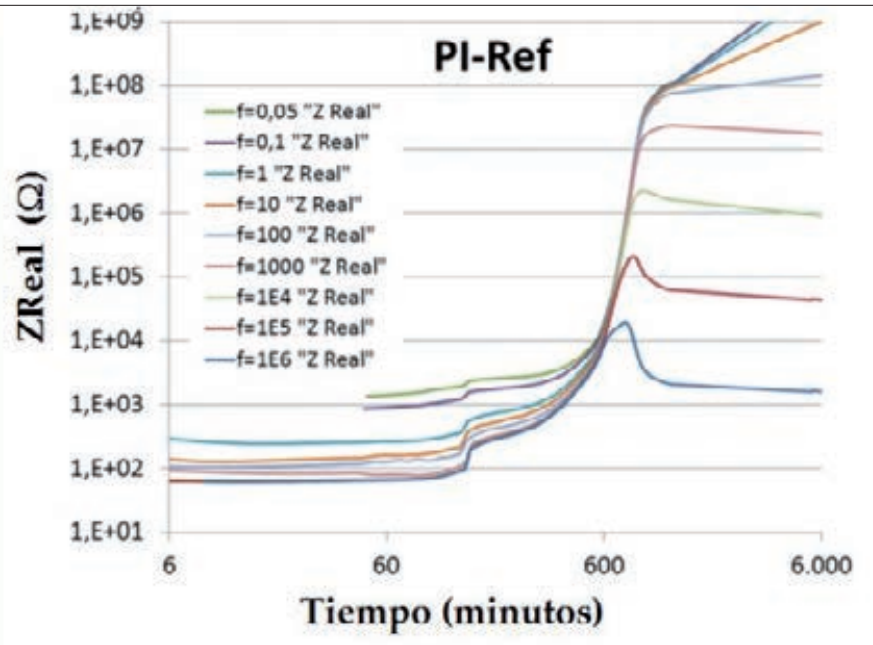

(b)

Figura 6. Evolución con el tiempo de hidratación para el material de referencia (PI-Ref), a diferentes frecuencias (a) Módulo de la admitancia y (b) parte real de la impedancia.

relacionarlo con la formación completa de las fases cristalinas del material una vez hidratado, se produce una disminución acusada de la impedancia y a partir de un cierto tiempo, se mantiene prácticamente estable. Esta disminución en el valor de la parte real de la impedancia, puede relacionarse con la incorporación del agua estructural en la composición del material, lo que pone de manifiesto que el material ya está conformado con una estructura cristalina que sufrirá pocas modificaciones con el tiempo.

En la figura 7 se compara la respuesta para los diferentes materiales analizados. De acuerdo con lo comentado anteriormente, se representa a bajas frecuencias la evolución del módulo de la admitancia (figura 7a), y para altas frecuencias, la parte real de la impedancia (figura $7 \mathrm{~b}$ ). En todos los casos se observa un primer incremento en la admitancia indicando el proceso de inicio de la hidratación, y la presencia de iones libres en el mismo. Posteriormente tiene lugar la disminución de la admitancia a medida que se van formando las nuevas estructuras cristalinas. El proceso de transformación tiene lugar primero para el FAL25, prácticamente en la primera hora de hidratación, le sigue el BAC25, que se retrasa aproximadamente 25 minutos, y prácticamente una hora después tiene lugar en BAL25, PI-Ref, FAC25, FBAL, y por último FBAC. Se observa también que el proceso de transformación sigue ritmos diferentes para cada uno de compuestos, con un proceso muy brusco en los que primero tienen la transformación, y con una ralentización y continuidad en el tiempo de la transformación a medida que se retrasa su inicio. Si completamos el análisis haciendo uso de la variación de la parte real de la impedancia, y asociando el máximo de la impedancia con el hidratado del material y la formación de la estructura cristalina final del material, observamos cambios significativos. El compuesto que completa antes su transformación es el material de referencia. Aunque el proceso para este compuesto se inicia prácticamente una hora más tarde que para el FAL, tiene lugar de forma mucho más rápida llegándose al máximo de ZReal a las 12 horas. Es curioso el comportamiento del FAL, que tiene un primer proceso de estabilización de las fases cristalinas, y

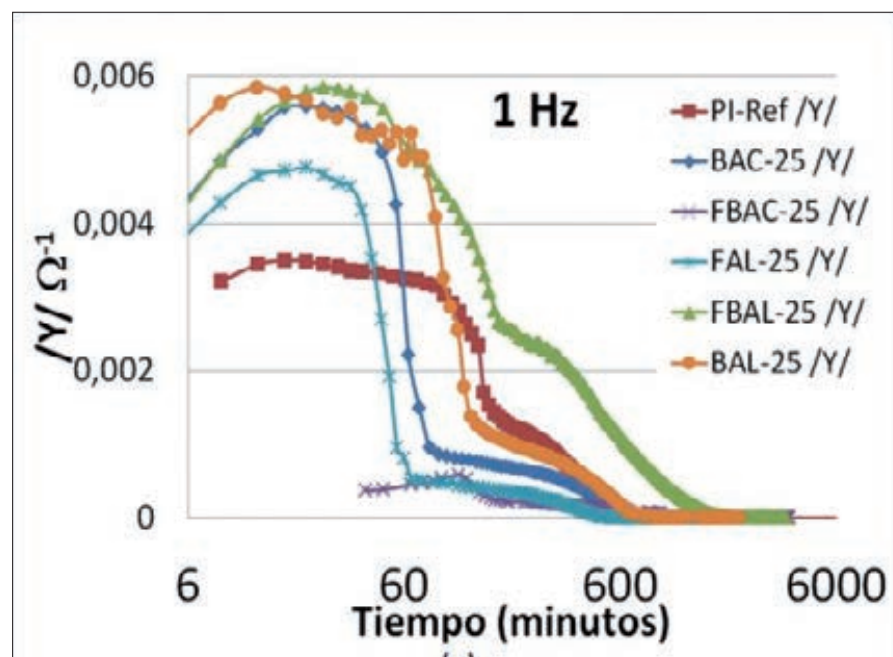

(a)

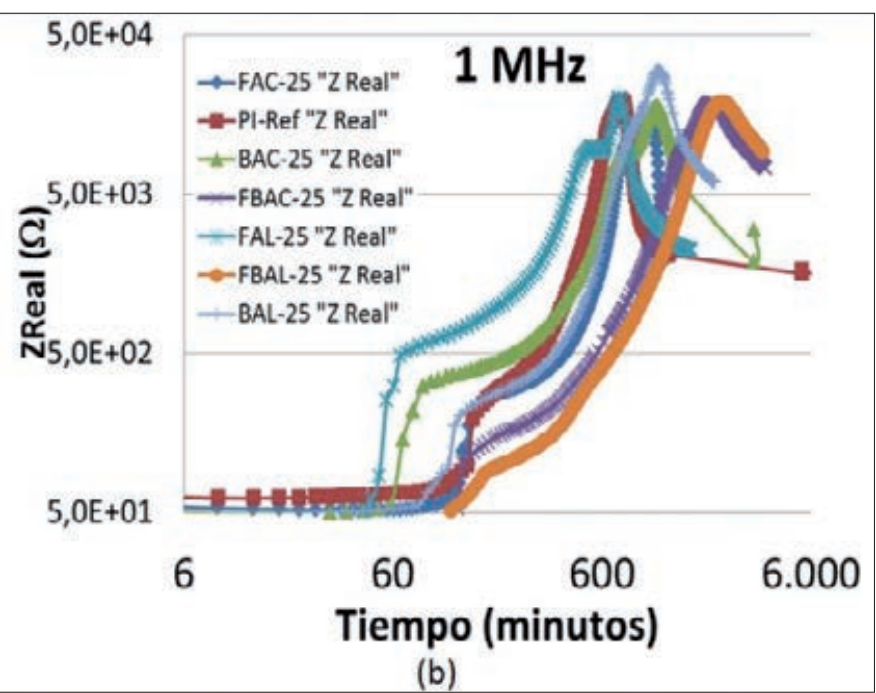

(b)

Figura 7. Comparación entre los diferentes compuestos de su evolución con el tiempo de hidratación (a) Módulo de la admitancia medido a 1 Hz y (b) parte real de la impedancia medido a $1 \mathrm{MHz}$. 


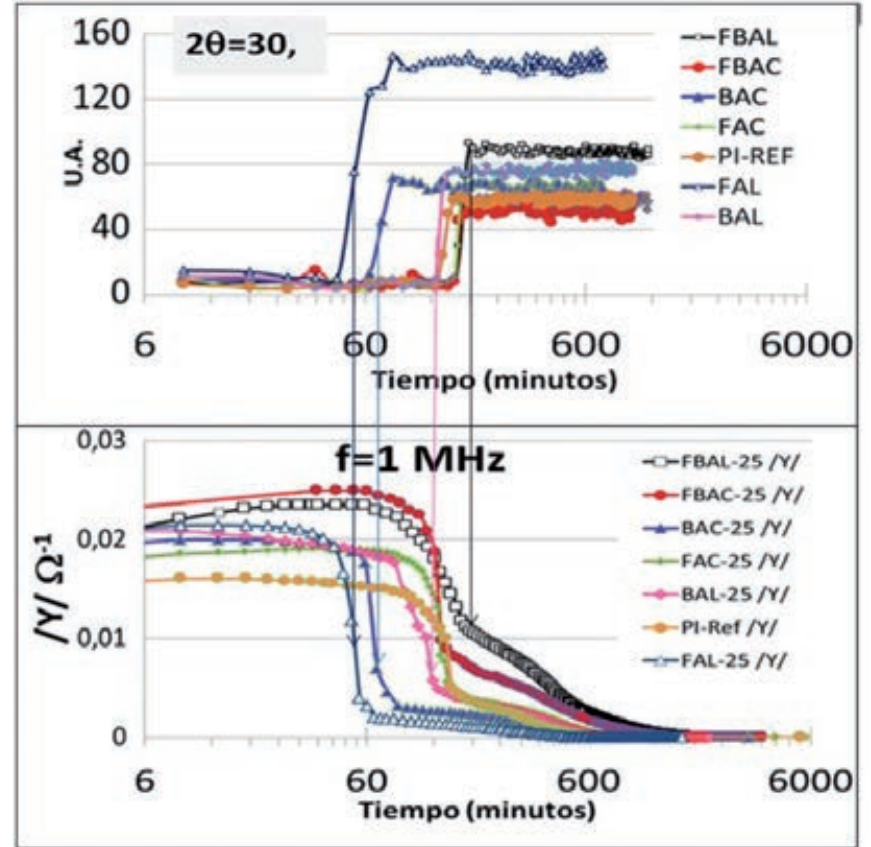

Figura 8. Comparación del análisis temporal analizado por a) DRX, con el análisis de la evolución del pico de $2 \theta=30,96$ y b) EIS, con la representación del módulo de la admitancia medido a $1 \mathrm{MHz}$ de frecuencia.

posteriormente se reactiva el proceso, completándose tanto en magnitud como en comportamiento como en tiempos de forma muy similar al del material de referencia. De forma similar, aunque los procesos se inician en tiempos diferentes, se completan prácticamente a la vez para los compuestos BAL, FAC y BAC (máximo aproximadamente a las 16 horas). Por último, los compuestos que incluyen tanto cenizas como escorias (FBAC y FBAL) son la que tardan más en completar el proceso (mínimo 34 horas).

Si comparamos los resultados obtenidos para el análisis de los máximos de DRX con la respuesta de los diferentes parámetros eléctricos, encontramos una excelente concordancia entre ambos. En la figura 8 se compara los tiempos de inicio de las transformaciones analizados a partir de DRX y EIS. En este caso el parámetro eléctrico que se utiliza es el módulo de la admitancia pero medido a altas frecuencia $(\mathrm{MHz})$ para relacionar la respuesta con las características intrínsecas del material. Si se compara este resultado con el recogido en la figura $7 \mathrm{a}$, puede verificarse que el inicio de la transformación tiene lugar de forma más brusca en todos los casos. Esto es debido a que a estas frecuencias, parte de los iones presentes en el material no son capaces de seguir el cambio de frecuencia, y no contribuyen a la variación de la impedancia. Con las flechas se indican las equivalencias entre los comportamientos de los diferentes compuestos para los dos procedimientos de estudio.

\section{CONCLUSIONES}

Las escorias resultantes de la combustión de carbón en centrales térmicas tienen una composición química similar al de las cenizas volantes excepto en hierro, manganeso y cromo, que es mayor.
Los distintos tipos de adiciones modifican los tiempos en el proceso de hidratación consiguiéndose en todos los casos la estructura cristalina característica del mismo.

El seguimiento en el tiempo de los picos de DRX permite determinar el inicio y final de diferentes procesos. En una primera aproximación al análisis por DRX, puede concluirse que el utilizar un tipo $\mathrm{u}$ otro de residuos puede adelantar o atrasar el proceso de hidratación.

Las medidas de EIS son una potente herramienta de análisis, aportando la ventaja de poder aplicarse en obra. La monitorización del módulo de la admitancia resalta los cambios que tienen lugar a tiempos cortos (inferiores a las 5 horas) y el módulo de la impedancia real lo hace para tiempos largos. El uso de diferentes frecuencias de análisis permite visualizar la movilidad de cargas (hasta $10 \mathrm{kHz}$ ) o la estructura interna del material (frecuencias superiores a 100 $\mathrm{kHz}$ ). Además permite determinar con facilidad el inicio del proceso, y el tiempo en que se completa.

Respecto al inicio del proceso de hidratación, existe una muy buena concordancia entre las medidas eléctricas y de difracción de Rayos-X.

Se pone de manifiesto que el proceso de hidratación se inicia primero en el FAL25, prácticamente en la primer hora de hidratación, le sigue el BAC25, que se retrasa aproximadamente 25 minutos, y prácticamente una hora después tiene lugar en BAL25, PI-Ref, FAC25, FBAL, y por último FBAC.

Mediante EIS, se observa también que el proceso de transformación sigue ritmos diferentes para cada uno de compuestos, con un proceso muy brusco en los que primero tienen la transformación, y con una ralentización y continuidad en el tiempo de la transformación a medida que se retrasa su inicio. El compuesto que completa antes su transformación es el material de referencia. Aunque el proceso para este compuesto se inicia prácticamente una hora más tarde que para el FAL, tiene lugar de forma mucho más rápida llegándose al máximo de ZReal a las 12 horas. Es curioso el comportamiento del FAL, que tiene un primer proceso de estabilización de las fases cristalinas, y posteriormente se reactiva el proceso, completándose tanto en magnitud como en comportamiento como en tiempos de forma muy similar al del material de referencia. De forma similar, aunque los procesos se inician en tiempos diferentes, se completan prácticamente a la vez para los compuestos BAL, FAC y BAC (máximo aproximadamente a las 16 horas). Por último, los compuestos que incluyen tanto cenizas como escorias (FBAC y FBAL) son la que tardan más en completar el proceso (mínimo 34 horas).

\section{AGRADECIMIENTOS}

Este trabajo está financiado por el proyecto MAT201021088-C03-03 (España)

\section{BIBLIOGRAFÍA}

1. Siddique R.. (2010): Utilization of coal combustion by-products in sustainable construction materials. Resources, Conservation and Recycling, 54(12), pp. 1060-1066

2. Asokan P., Saxena M. and Asolekar S. R.. (2005): Coal combustion residues-environmental implications and recycling potentials. Resources, Conservation and Recycling, 43(3), pp. 239-262. 
3. Izquierdo M. and Querol X.. (2012): Leaching behaviour of elements from coal combustion fly ash: An overview. International Journal of Coal Geology, 94(0), pp. 54-66

4. ECOBA (2009): Production and Utilisation of CCPs in 2009 in Europe. Disponible en: http:/ / www.ecoba.org/.

5. Real Decreto 956/2008, de 6 de junio, por el que se aprueba la instrucción para la recepción de cementos (RC-08). Boletín Oficial del Estado, núm. 148, p.27794-27841.

6. Cheriaf M., Rocha J. C. and Péra J.. (1999): Pozzolanic properties of pulverized coal combustion bottom ash. Cement and Concrete Research, 29(9), pp. 1387-1391.

7. Jaturapitakkul C. and Cheerarot R.. (2003): Development of Bottom Ash as Pozzolanic Material. Journal of Materials in Civil Engineering, 15(1), pp. 48-53.

8. H. Kurama and M. Kaya. (2008): Usage of coal combustion bottom ash in concrete mixture. Construction and Building Materials, 22(9), pp. 19221928 .

9. Kula I., Olgun A., Erdogan Y. and Sevinc V.. (2001): Effects of colemanite waste, cool bottom ash, and fly ash on the properties of cement. Cement and Concrete Research, 31(3), pp. 491-494.

10. Kula I., Olgun A., Sevinc V. and Erdogan Y.. (2002): An investigation on the use of tincal ore waste, fly ash, and coal bottom ash as Portland cement replacement materials. Cement and Concrete Research, 32(2), pp. 227-232.

11. Canpolat F., Yılmaz K., Köse M. M., Sümer M. and Yurdusev M. A.. (2004) Use of zeolite, coal bottom ash and fly ash as replacement materials in cement production. Cement and Concrete Research, 34(5), pp. 731-735.

12. C Argiz. Menendez. E. and Sanjuán M. A.. (2013): Effect of mixes made of coal bottom ash and fly ash on the mechanical strength and porosity of Portland cement. Materiales de Construcción. 63 (309), 49-64

13. Taylor H. F. W. (1997): Cement Chemistry. London: Thomas Telford.

14. Gordana S., Ljubica C., Živko S. and Srdan M.. (2007): Hydration study of mechanically activated mixtures of Portland cement and fly ash. Journal of the Serbian Chemical Society, 72(6), pp. 591-604.

15. EN 196 - 4 (2005): Method of cement tests. Physical test. Determination of hydration heat by semi-adiabatic calorimetry (Method of Langabant calorimetry).

16. Gartner E. M., Young J. F., Damidot D. A. and Jawed I.. (2002). Hydration of portland cement. In J. Bensted and P. Barnes (Eds.), Structure and performance of cements (Second ed), pp. 57-113

17. Xiao L. and Li Z.. (2009): New Understanding of Cement Hydration Mechanism through Electrical Resistivity Measurement and Microstructure Investigations. Journal of Materials in Civil Engineering, 21, pp. 368-373.

18. Tamás F. D., Farkas E., Vörös M. and Roy D. M.. (1987): Low-frequency electrical conductivity of cement, clinker and clinker mineral pastes. Cement and Concrete Research, 17(2), pp. 340-348

19. Levita G., Marchetti A., Gallone G., Princigallo A. and Guerrini G. L. (2000): Electrical properties of fluidified Portland cement mixes in the early stage of hydration. Cement and Concrete Research, 30(6), pp. 923-930.

20. Menéndez E. and de Frutos J.. (2009): Ion monitoring solutions in cements using electrical spectroscopy. Revista Mexicana de Física, 55(1), pp. 76-80.

21. Menéndez E. and de Frutos J.. (2011): Equivalencia entre medidas eléctricas y difracción de rayos $X$ en la formación de fases cristalinas de pastas de cemento. Boletín de la Sociedad Española de Cerámica y Vidrio, 50, pp. 225-234.
22. Menéndez E. and de Frutos J.. (2010): Sequential Characterization of the Hydration Products of Cements with Additions by a Combination of Destructive and Non-Destructive Techniques: Calorimetry, XRD, AFM and EIS. MRS Proceedings, 1275.

23. Raupp-Pereira F., Ribeiro M. J., Segãdes A. M. and Labrincha J. A. (2007): Setting behaviour of waste-based cements estimated by impedance spectroscopy and temperature measurements. Boletín de la Sociedad Española de Cerámica y Vidrio, 46(2), pp. 91-96.

24. de Frutos J., Matutes-Aquino J. A., Cebollada F., Botello-Zubiate M. E. and Menéndez E.. (2007): Synthesis and characterization of electroceramics with magnetoelectric properties. Journal of the European Ceramic Society, 27, pp. 3663-3666.

25. Andrade C., Blanco V. M., Collazo A., Keddam M., Nóvoa X. R. and Takenouti H.. (1999): Cement paste hardening process studied by impedance spectroscopy. Electrochimica Acta, 44(24), pp. 4313-4318.

26. de Frutos J., Soler L. and Andrade C.. (1999): Propiedades dieléctricas de pastas de cemento con reducido contenido en agua libre. Boletín de la Sociedad Española de Cerámica y Vidrio, 36(6), pp. 607-610.

27. Cabeza M., Keddam M., Nóvoa X. R., Sánchez I. and Takenouti H.. (2006) Impedance spectroscopy to characterize the pore structure during the hardening process of Portland cement paste. Electrochimica Acta, 51(8-9), pp. 1831-1841.

28. Taylor M. A. and Arulanandan K.. (1974): Relationships between electrical and physical properties of cement pastes. Cement and Concrete Research, 4(6), pp. 881-897.

29. Gu P., Xu Z., Xie P. and Beaudoin J. J.. (1993): Application of A.C impedance techniques in studies of porous cementitious materials: (I) Influence of solid phase and pore solution on high frequency resistance. Cement and Concrete Research, 23(3), pp. 531-540.

30. Menéndez E. and de Frutos J.. (2012): Generación y caracterización de microgrietas en materiales estructurales. Boletín de la Sociedad Española de Cerámica y Vidrio, 51, pp. 45-54.

31. Menéndez E. and de Frutos J.. (2009): Análisis no destructivo del estado de degradación en morteros bajo la acción de ciclos hielo-deshielo. Boletín de la Sociedad Española de Cerámica y Vidrio, 48, pp. 223-230.

32. Cruz J. M., Fita I. C., Soriano L., Payá J. and Borrachero M. V.. (2013): The use of electrical impedance spectroscopy for monitoring the hydration products of Portland cement mortars with high percentage of pozzolans. Cement and Concrete Research, 50(0), pp. 51-61.

33. Dotelli G. and Mari C. M.. (2001): The evolution of cement paste hydration process by impedance spectroscopy. Materials Science and Engineering: A, 303(1-2), pp. 54-59.

34. UNE - EN 196 - 2 (2006): Métodos de ensayo de cementos. Parte 2: análisis químico de cementos. Madrid: AENOR.

35. UNE - EN 197 - 1 (2011): Métodos de ensayo de cementos. Parte 1: Composición, especificaciones y criterios de conformidad de los cementos comunes. Madrid: AENOR.

Recibido: 06/09/2013

Recibida versión corregida: 16/12/2013

Aceptado: 18/12/2013 